\title{
An Overview of Exclusive Pharmacotherapeutical Agents and Aids Used in Dental Field
}

Dr. R. Shivani ${ }^{*}$, Dr. Satish Vasanth ${ }^{2}$, Dr. Sharmila. $\mathbf{M}^{3}$, Dr. U. Vivekanandan ${ }^{4}$

${ }^{1}$ Assistant Professor, Department of Dentistry, Madha Medical College and Research Institute, Chennai, India.

E-mail: drshivanirmds@gmail.com

2Senior Lecturer, Department of oral and maxillofacial surgery Madha Dental College and Hospital, Chennai, India.

E-mail: vasanthsmile@gmail.com

3Post Graduate Student, Department of Orthodontics and Dentofacial Orthopedics, Thai Moogambigai Dental College and Hospital, Chennai, India.

E-mail: sharmisharmila84@gmail.com

${ }^{4}$ Senior Lecturer, Department of Orthodontics and Dentofacial Orthopedics, Thai Moogambigai

Dental College and Hospital, Chennai, India.

E-mail: vivekanandhan.umapathy@gmail.com

Review Article

Address for Correspondence Author

Dr. R. Shivani, Assistant Professor, Department of Dentistry, Madha Medical College and Research Institute, Chennai, India.

E-mail: drshivanirmds@gmail.com

Crossref doi: https://doi.org/10.36437/ijdrd.2020.2.1.L

\section{ABSTRACT}

Treatment procedures in dentistry involve a lot of pharmacotherapeutical agents and aids along with medications. Certain pharmacotherapeutical products are designed and manufactured exclusively for its use in dentistry. These dental therapeutic agents and aids are left unspoken and needs attention as their use in dentistry is high. Both the dentist and the pharmacist should be aware of these products and should update themselves on the newer products available in the market. This article emphasizes on such exclusive products which are used in dentistry.

Keywords: Pharmacotherapeutics, Dental treatment, Dental practice, Dental Field

\section{Introduction}

Dental practice and pharmacotherapy are very much inseparable which goes hands in hands. Dentistry is a huge branch which allows treating the dental ailments not only with medications but also with various other therapeutic agents, dental materials, mechanical aids and techniques. Though the use of drugs and other pharmacological agents are common in dentistry, there are some exclusive 
pharmacotherapeutical agents and aids in this field which are left unspoken and needs attention. The dental practitioner expects these products to be readily available in the pharmacy, as they are prescribed to the patients. Moreover dentists have a moral duty to deliver quality oro-dental healthcare with the pharmacotherapeutical agents.[1] This article emphasizes on those agents which are designed and manufactured for dental use only.

\section{Exclusive Dental Pharmacotherapeutical Agents and Aids}

Dentistry uses various pharmacological agents such as anesthetics, antiseptics, analgesics, antibiotics, muscle relaxants, steroids, antimicrobials, antifungals, antivirals, antianxiety drugs.[2] Hyaluronidase injection, placental extracts, anti oxidants, vitamin and micronutrients supplements are also used frequently in dentistry for treating various oral conditions. Along with these agents, certain therapeutic agents which are designed for its exclusive use in dentistry are also commonly employed in dental practice. These agents and aids play a vital role in the dental treatment modalities. Dentists prescribe these products to their patients as a part of dental treatment. Various dental pharmacotherapeutical products are discussed below.

\section{Therapeutical Agents and Aids}

\section{Saliva Associated}

a. Sialogogues: Sialogogue is a drug or substance which activates muscarinic cholinergic receptors of the parasympathetic nervous system that increases the flow rate of saliva. They are used in the treatment of xerostomia. Xerostomia occurs from various diseases like sjogren's syndrome, diabetes insipidus, pernicious anemia, rheumatoid arthritis, as adverse effect of drugs, radiation, post cancer therapy or as a result of aging. Sialogogues stimulate any functioning salivary gland tissue to produce increased amount of saliva. They are supplied in the form of oral sprays, gels, chewing gums, lozenges, solutions. ${ }^{22]}$ Pilocarpine and cevimeline are two US Food and Drug Administration-approved systemic sialogogues for treatment of dry mouth. Sugar-free chewing gums induce stimulated saliva secretion of the minor salivary glands in the oral cavity.[3] Topical Mucosal sialogogue sprays containing $1 \%$ malic acid is given for patients with antidepressant- or antihypertensiveinduced dry mouth. Oral spray containing oxygenated glycerol tri-ester is also preferred. In these oral sprays, water, glycerin, sorbitol, mannitol, xylitol, ascorbic acid, citric acid are used in various compositions. ${ }^{[3]}$ Oral solutions are available for stimulating saliva and contains dibasic and monobasic sodium phosphate, sodium chloride, calcium chloride, sodium carboxymethylcellulose, glycerin are mixed in various compositions and added flavours. Different commercially available saliva stimulating lozenges and gels are also used in treatment of dry mouth. Commercially available sialogogues are Xylitol chewing gums, Wet fast lozenges, Xerostat spray, Salistat tablets etc.

b. Antisialogogues: Antisialogogues decrease the salivary secretion by cholinergic antagonist action. They inhibit the action of salivary gland myo-epithelial cells. The synthetic atropine derivatives, propantheline and methantheline are few examples of anti-sialogouges. Several other drugs including opium, belladonna have the same action.[2] Antisialogogues are used before commencing dental treatments like bonding, orthodontic bracketing, prosthetic procedures, impression taking, restorative procedures, radiographic procedures when a dry field is required.[4] One of the commercially available antisialogogues are Pro- 
Banthine $15 \mathrm{mg}$ tablets which should be given 30 minutes before commencing the dental procedure. Most of the antisialogogues has its duration of action from 4 to $6 \mathrm{hrs}$.

c. Saliva Substitutes: Saliva substitutes aim to increase viscosity and mimic natural saliva without altering the salivary flow. These agents contain carboxymethylcellulose or hydroxyethylcellulose, minerals such as fluoride, calcium, and phosphate ions, flavoring agents, and preservatives (eg, propyl or methyl paraben). Carboxy methyl cellulose based artificial saliva demonstrated moderate effects in reducing dry mouth related symptoms with more significant effects appearing in patients whose residual secretory potency was severely compromised.[2] Commercially available artificial saliva brands are Moi-stir artificial saliva, Sarsaliva, Aquasub artificial saliva spray etc.

\section{Gingiva Associated}

a. Gum Paint: Gum paints are the combination of antiseptics and tanning agents which precipitate proteins but do not penetrate cells thereby affecting only the superficial layer making it mechanically stronger and decreases exudation. They have germicidal, fungicidal, anesthetic and healing properties. They give a soothing, cooling and an astringent effect on application. They contain Choline salicylate, Tannic acid, Cetrimide, Thymol, Camphor, Cinnamon oil, Iodine and Alum (hydrated potassium aluminium sulfate).[2] Patients are advised to apply on the affected area several times with the cotton applicator or with finger for the treatment of stomatitis, inflammation and bleeding gums. It also decreases sensitivity and increases gingival resistance against infections. [5] Commercially available gum paints are Sensoform gum paint, Stolin-R gum paint, Astrigum gum paint.

b. Gum Stimulator: The gum stimulator is a device used to massage the gingival tissues thereby promoting the blood flow. It has been designed to promote healthy and firm gingiva by massaging and activating the gingival microcirculation. Mechanical stimulation of gingiva is a traditional method to maintain gums in good health. Gum stimulator is available as a separate device with handle with a rubber or silicone tip, electric gum massager with a rubber tip or attached with the tooth brush. Finger gum massager is available as rubber tips which can be worn in fingers. The replacement tips are also sold separately. [6] Commercially available gum stimulators are Gum Butler, Flossaid gum massager. Replacement tips are available commercially as Sunstar Gum stimulator refills.

\section{Denture Related}

a. Denture Cleansers: Denture cleansing agents are the products designed to remove stains, deposits, and debris from the surfaces of dental prostheses. They are available in the form of creams, gels, pastes, solutions and tablets. These agents consist of disinfectants, detergents, chelating agents, oxidizing and reducing agents. The denture cleansing creams, pastes and gels are used to brush the denture and then rinsed off. Denture cleansing tablet is immersed in warm water and then the denture is soaked and brushed with that solution. Denture cleansing solution is used to soak the denture for few minutes to overnight according to manufacturer's instruction. They are used only after removing the denture from the mouth. [7] Commercially available denture cleansers are Fittydent cleansing tablet, Clinsodent powder, Polident etc. 
b. Denture Adhesive: Denture adhesives are materials used to increase denture retention. This improves chewing ability thereby improving comfort and confidence of the patient. It reduces denture wobble and also the amount of food particles accumulation under the dentures. They are commercially supplied as denture adhesive paste, powder and cream. ${ }^{8]}$ They are composed of adhesive agents (tragacanth, gelatin, methyl-cellulose, acacia, hydroxyl-methyl cellulose, Karaya gum, synthetic polymers), antimicrobial agents (Sodium tetraborate, ethanol, hexachlorophene, sodium borate) plasticizing agents, flavoring agents and wetting agents.[9] Commercially available denture adhesives are Fixon, Denofit etc.

\section{Chewing Gums}

It is one of the most common confectionary products consumed worldwide. In dentistry, it is advised for various other reasons. Dental use of chewing gums are as nicotine substitutes (Eg: Nicotex), desensitizing chewing gums for post bleaching tooth sensitivity (Eg: GC Recaldent chewing gum) and as saliva stimulating chewing gums (Eg: Wrigley's Orbit). Dentists have a moral duty to counsel for tobacco cessation hence they advice their patients to use nicotine substituting chewing gums as a part of nicotine replacement therapy.[10] It consists of gum base, softeners, sweeteners, and flavouring agents. Chewing gum is now recognized as a legitimate oral drug-delivery dosage form hence various drug companies have recognized such delivery systems and medicated chewing gums are available in market.[11]

\section{Oral Topical Anesthetic}

Oral topical anesthetics act on the peripheral nerves and provide anesthetic effect at the site of application. They contain lidocaine or benzocaine. This may also be available in combination with other analgesic medications. They are available as solutions, creams, gels, and sprays. It reduces the local pain caused by dental treatment procedures like needling, placement of orthodontic bands, periodontal treatment procedures, sub gingival scaling, rubber-dam clamp placement etc. It also reduces vomiting reflex, oral mucositis, oral ulcers. They reduce dental phobia especially in children by alleviating discomfort and pain. ${ }^{[12]}$ Commercially available oral topical anesthetics are Mucopain gel, Dentogel etc.

\section{Oral Topical Corticosteroid}

Oral topical corticosteroid provides excellent anti-inflammatory and immuno-modulatory properties in treating mild to moderate symptomatic oral lesions. They are available as gel, pellets, paste, lozenges and orabase. They are widely used in the therapeutics of oral lichen planus, recurrent apthous stomatitis, erythema multiforme, pemphigus, mucous membrane pemphigoid, bullous pemphigoid, systemic lupus erythematosus etc. It reduces inflammation and pain in such conditions when applied topically. [13] Kenacort, Oraways buccal paste are few commercially available oral topical corticosteroids.

\section{Oral topical Antifungal}

These are the first line of treatment for superficial oral fungal infections. They show fungicidal or fungistatic actions with fewer side effects. Unlike oral drugs topical applications avoids drug to drug interactions. Topical antifungal agents are available as rinses and gels. Clotrimazole, nystatin, itracanazole, micanazole, flucanazole are few antifungal drugs used as topical agents. 
[14] Other oral topical drugs include topical application of antibiotics, antivirals, neuropathics, immunomodulators, chemopreventive agents and counter irritants. Commercially available oral topical antifungals are Candid, Nuforce mouth paint etc.

\section{Tooth Desensitizing Gels}

Dentists prescribe tooth desensitizing gels as at-home treatment for patients with dentinal hypersensitivity. Topical casein phosphopeptide-amorphous calcium phosphate is supplied in the form of topical gels which are applied over the tooth surface. The peptides bind to the dentin surface and promote the deposition of mineral deposits within dentin tubules. ${ }^{[15]}$ Tooth mousse, GLUMA desensitizer, Bexident gel are few commercially available desensitizing gels.

\section{At-home Bleaching Kit}

At-home bleaching kit is a kit advised by the dentist to the patient who seeks for teeth whitening. It consists of a mouth guard like tray and peroxide based bleaching gel or paste. This may be performed alone by the patient at home or along with in-office bleaching. ${ }^{[16] ~ L u m i b r i t e}$ take home whitening system from DenMat, Opalescence PF take home dental bleaching from Ultradent are few commercially available at-home bleaching kits.

\section{Oral Hygiene Agents and Aids}

1. Tooth Paste: Tooth pastes are daily oral care products when used with tooth brush for mechanical cleaning of the teeth. They are classified as;

a. Fluoride Tooth Paste: Toothpaste used as a local fluoride source to inhibit the development of caries. According to concentration of fluoride present, they are classified as tooth paste containing fluoride concentration upto $1000 \mathrm{ppm}$, between 1000-1500 ppm and 2500-5000 ppm. ${ }^{[17]}$ Colgate total fluoride tooth paste, Oral-B fluoride tooth paste, Amflor organic fluoride tooth paste are few commercially available well known brands of fluoride tooth pastes.

b. Periodontal Tooth Paste: Tooth pastes meant for patients with periodontal disease by removing plaque and preventing bacterial growth. It can be composed of natural plant extracts, essential oils, enzymes or vitamins or with synthetic antiseptic or antibacterial substances such as triclosan, chlorhexidine, hydrogen peroxide, baking soda, Povidone Iodine, zinc citrate. Periodontal tooth pastes are used for prevention and treatment of periodontal disease. [17] Parodontax, Colgate gum care, Sensodyne gum care are few commercially available periodontal tooth paste brands.

c. Desensitizing Tooth Paste: Antisensitivity tooth pastes are preferred for patients who suffer from dentinal hypersensitivity. These tooth pastes are classified according to their mode of action as analgesic tooth paste with potassium nitrate as main component and dentinal tubule blocking tooth paste with stannous fluoride as major ingredient.[17] Elsenz, Hydent pro, Shy NM, Thermoseal, Sensodent-K are few desensitizing tooth pastes.

d. Esthetic Tooth Paste: These tooth pastes are meant for correcting the dental esthetics of the patient. It can be classified as whitening tooth paste and bleaching tooth paste. Whitening tooth paste aims in removing plaque as it contains abrasives. Bleaching tooth paste contains chemicals, most commonly hydrogen peroxide or calcium peroxide and provides a bleaching effect to the tooth surface.[17] Sensodyne pronamel gentle whitening tooth paste, Colgate visible 
white tooth paste, Crest 3D white brilliance are few whitening and bleaching tooth paste brands.

e. Tooth Pastes with a Specific Purpose: Certain tooth pastes are meant for specific functions. For example, xerostomia can be treated with toothpastes containing olive oil, betaine and xylitol which will stimulate salivary secretion.[17] Biotene orabalance moisturing gel is a commercially available brand for dry mouth cases.

f. Kids Tooth Paste: There are tooth pastes designed for children and are advised with safe brushing and spitting practices. They are artificially flavored and colored with fluoride releasing property. This can increase positive oral health habits among children.[18] The fluoride content in these tooth pastes vary from $500 \mathrm{ppmF}$ to $1,450 \mathrm{ppmF}$. Fluoridated tooth pastes are not recommended for children under 2 years of age owing to the risk of fluorosis.[19] Colgate kids tooth paste, Kidodent, Pediflor kids tooth paste are few commercially available kids tooth paste brands.

2. Tooth Brush: Tooth brush is a mechanical cleansing aid used for routine oral care. Well known manufacturers like Colgate and Oral-B manufacture all these type of tooth brushes discussed below.

a. Manual Tooth Brush: They are designed in different shapes of brush head, bristles arrangement and handle designs.

b. Electric Tooth Brush: Electric tooth brushes are mainly developed for patients with manual dexterity problems or other physical limitations. They can be of two types: battery operated and powered. The bristles will spin, move back and forth in a gentle pattern when it is fully charged and switched on. [20] Sonic and ultra sonic tooth brushes produce high frequency vibrations (1.6 MHz) and aids in stain removal and disruption of the bacterial cell wall. [21]

c. Tooth Brush for Specific Purposes: There are tooth brushes designed for specific purposes. Interdental tooth brush is a small brush used for cleaning interdental space or space between teeth and the wire of dental braces. Dentists will analyze the clinical condition and prescribe such brushes for their patients. End tufted tooth brush is ideal for cleaning specific inaccessible areas such as wisdom teeth and crooked teeth. Ortho tooth brushes are designed for cleaning teeth with orthodontic braces. Patients wearing ortho appliances are prone for plaque accumulation and hence a specifically designed mechanical cleaning aid is required. [21] Based on the bristle thickness, tooth brushes are classified as hard, medium, soft and ultrasoft, in which soft and ultrasoft tooth brushes are often prescribed by the dentists. Tooth brushes are designed also for kids in various sizes, colors and handle designs to attract them towards brushing.

3. Mouth Wash: These are solutions used as a safe and effective chemical oral hygiene agent. It reduces microbial load, masks bad breath, prevents plaque accumulation and reduces dental caries.[22] Varieties of brands of mouth wash are available in the market. Most of them contain alcohol, chlorhexidine gluconate and triclosan. Owing to the side effects of alcoholic mouth washes such as xerostomia, burning mouth, taste alterations, non-alcoholic mouthwashes were developed. Presently herbal mouthwashes which are derived from various herbs and plant extracts have been introduced.[23][24] Commercially available mouthwashes are Listerine, 
Rexidine, Colgate plax, Closys. Currently prominent manufacturers like Colgate, Listerine, Himalayas are manufacturing mouthwashes with herbal extracts.

4. Dental Floss: Dental floss is a very fine thread that is often waxed to allow it to slide between adjacent teeth. They may be colored and flavored. The types are waxed and unwaxed floss. Flossing removes dental plaque from the interproximal areas of teeth which are inaccessible for tooth brush. Floss can be used along with flossing tools such as floss holders which hold floss securely in place and floss threaders which help thread floss in tight spaces such as around a fixed bridge. Oral irrigators also called as dental water jets or water flossers helps in cleaning between teeth and around dental implants and bridges. [25] Johnson \& Johnson Reach dental floss, Colgate dental floss, Oral-B dental floss are few commercially available products.

Other than these agents, several other dental pharmacological agents including periodontal dressing, gingival retraction cord, periodontal local drug delivery systems and various restorative materials also have professional applications in dentistry. Diagnostic agents such as plaque disclosing solution which is used to detect plaque deposition, toluidine blue solution and lugol's iodine solution which are helpful in oral cancer screening and early diagnosis are also included. These agents will be available from a dental supplier.

\section{Recent Advances in Dental Pharmacotherapeutics}

Caries Vaccine in Research: Dental caries is an infectious disease and one of the most common diseases occurring in humans. Although different caries preventive approaches currently exist, development of an efficient vaccine has been studied for more than three decades. Though animal trials with caries vaccine showed promising results, few human trials ended up with significant difference of opinions. The main challenge in developing dental caries vaccine is to remove human heart cross reactivity and risk of rheumatic fever from any future caries vaccines. Scientists are still working on developing a safer caries vaccine and are cautiously optimistic that dental caries vaccine will be available sooner for universal human utilization.[26]

\section{Conclusion}

Pharmocotherapeutical agents and aids play a major role in dental field. Many newer products are still developing and the available products are still be modified by the manufacturers to ease their use. Both the dentist and the pharmacist should have a thorough knowledge regarding these exclusive products used in dentistry. They should update themselves on the newer products available in the market. This article is an attempt to join the gap between dental practice and pharmacotherapeutics, aiming to encourage more research papers in the field of pharmacotherapeutics as significant in dentistry.

\section{References}

1. Patil K. Pharmacotherapeutics in dentistry. J Adv Clin Res Insights 2015;2:229-230.

2. Mohan M, Gupta A, Shenoy V, Parolia A. Pharmacological Agents in Dentistry: A Review. British Journal of Pharmaceutical Research 2011; 1(3): 66-87.

3. Villa A, Connell C L, Abati S. Diagnosis and management of xerostomia and hyposalivation. Therapeutics and Clinical Risk Management 2015;11:45-51. 
4. Kuijpers M A R, Vissink A, Ren Y, Kuijpers-Jagtman A M. The effect of antisialogogues in dentistry A systematic review with a focus on bond failure in orthodontics JADA 2010;141(8):954-965.

5. Gupta G et al. Astringents in dentistry: a review. Asian Journal of Pharmaceutical and health sciences. 2012;2(3):428-32.

6. Rumi Tano. Literature Review of Gingival Massage. Adv Dent \& Oral Health. 2018; 7(3): 555713.

7. Jain S G, Magdum D, Karagir A, Pharane P. Denture Cleansers: A Review. Journal of Dental and Medical Sciences 2015;14(2):94-96.

8. Yadav A, Yadav S. Denture adhesives-their stand in prosthodontics. The Journal of Indian Prosthodontic Society $2005 ; 5(2): 62-64$.

9. Kumar P R, Shajahan P A, Mathew J, Koruthu A, Aravind P, Ahammed M F. Denture Adhesives in Prosthodontics: An Overview. Journal of International Oral Health 2015; 7(1):93-95.

10. Sharma G, Puranik MP, Sowmya KR. Nicotine Replacement Therapy in Dental Settings: An Exploratory Survey in Bangalore City, India. Addict Health 2016; 8(1) 25-32.

11. Ahmed Mujib B R, Grover S, Vinayak V, Mittal S, Kumar M. Chewing Gum and Oral Health. Indian Journal of Contemporary Dentistry 2013;1 (1):72-74.

12. Hyo-seol Lee. Recent advances in topical anesthesia. J Dent Anesth Pain Med 2016;16(4):237244.

13. Sanghavi J, Aditya A. Applications of Corticosteroids in Dentistry. J Dent Allied Sci 2015;4:1924.

14. Shastry SP, Sanjay CJ, Kaul R, Mahima VG, Doggalli N. Topical drug delivery: An essential aid in the management of oral diseases. J Adv Clin Res Insights 2015;2:269-275.

15. Laurence J. Walsh. The effects of GC Tooth Mousse on cervical dentinal sensitivity: a controlled clinical trial. International Dentistry Sa;12 (1):4-12.

16. João Lima Rodrigues1, Patrícia Souza Rocha2, Silvia Letícia de Souza Pardim2, Ana Cláudia Vieira Machado1, André Luis Faria-e-Silva3, Paulo Isaías Seraidarian. Association Between InOffice And At-Home Tooth Bleaching: A Single Blind Randomized Clinical Trial. Brazilian Dental Journal (2018) 29(2): 133-139.

17. Ilze Maldupa, Anda Brinkmane, Inga Rendeniece, Anna Mihailova. Evidence based toothpaste classifi cation, according to certain characteristics of their chemical composition. Stomatologia, Baltic Dental and Maxillofacial Journal 2012;14:12-22.

18. Corey H. Basch \& William D. Kernan. Ingredients in Children's Fluoridated Toothpaste: A Literature Review. Global Journal of Health Science 2017;9(3):1-12.

19. Ellwood R P, Cury J A. How much toothpaste should a child under the age of 6 years use? European Archives of Paediatric Dentistry 2009;10 (3):181-187.

20. Aparna KS, Puranik MP, Sowmya KR. Powered tooth brush- a review. Int J Health Sci Res. 2018; 8(5):299-306.

21. Kumar G, Jalaluddin M D, Singh D K. Journal of Advances in Medicine 2013; 2(1): 65-76.

22. Akande 00, Alada ARA, Aderinokun GA, Ige AO. Efficacy of different brands of mouth rinses on oral bacterial load count in healthy adults. Afr J Biomed Res 2004;7:125-8.

23. Celso Augusto Lemos-Júnior, Germano Eduardo Miguel Villoria. Reviewed evidence about the safety of the daily use of alcohol-based mouthrinses. Braz Oral Res 2008;22(1):24-31. 
24. Bhat N, Mitra R, Reddy JJ, Oza S, Vinayak KM. Evaluation of efficacy of chlorhexidine and a herbal mouthwash on dental plaque: An in vitro comparative study. Int J Pharm Bio Sci 2013;4:625-32.

25. Joanna Asadoorian. Flossing. Canadian journal of dental hygiene 2006;40(3):1-10.

26. Giasuddin ASM, Huda SN, Jhuma KA, Haq AMM. Dental Caries Vaccine Availability: Challenges for the 21st Century. J Immuno Immuno the 2017;1: 002.

How to cite this Article: Dr. R. Shivani ${ }^{1 *}$, Dr. Satish Vasanth ${ }^{2}$, Dr. Sharmila. Mㄹ, Dr. U. Vivekanandan"; An Overview of Exclusive Pharmacotherapeutical Agents and Aids Used in Dental Field Int. J. Drug Res. Dental Sci., 2020; 2(1):5-13.

Source of Support: Nil, Conflict of Interest: None declared.

Received: 12-1-2020 Revised: 10-2-2020 Accepted: 25-2-2020 